International Journal of Engineering \& Technology, $7(1)(2017) 90-94$
SPC
International Journal of Engineering \& Technology
Website: www.sciencepubco.com/index.php/IJET
doi: $10.14419 /$ ijet. $v 7$ il. 8470
Research paper

\title{
Optimization tolerancing of surface in flexible parts and assembly: Influence Coefficient Method with shape defects
}

\author{
Atik Hafsa *, Chahbouni Mouhssine, Amegouz Driss, Boutahari Said \\ High School of Technology of Fez, University sidi mohamed ben abdellah B.P 2626 -Route d'Imouzzar, 30000 Fez, Morocco. \\ *Corresponding author E-mail: hafsa.atik@usmba.ac.ma
}

\begin{abstract}
Generally, a mechanical product must fulfill particular functions in accordance with the specification provided by the customer. A designer must find a solution with lower cost to answer the functional requirements. After the design stage, come the prototyping of the parts, but in case of the large structures of automotive and aeronautical parts, this step is impossible because of the large real dimension as well as the behavior of this type of parts during the positioning and during the assembly of all the mechanism.

The aim of this article is to show the influence of geometrical shape differences in the assembly of flexible components in order to optimize the tolerancing of surface in flexible parts and assembly. first a presentation of the tolerance of deformable mechanisms through the illustration of the general problem, then we propose a new approach which takes into account shape defects based on the Influence Coeficient Method, after that we compare between a case study without takes into account shape defects and another one but this time taking into account shape defects always based on the Influence Coefficient Method.
\end{abstract}

Keywords: Tolerance analysis, Deformable mechanisms, Influence Coefficients Method, surface contact, shape defects.

\section{Introduction}

The tolerancing for rigid parts may be inadequate when parts exhibit a high deformation. Automotive and aerospace industries, where this type of parts are very common. The high flexibility of such parts may cause wide shape variations during the assembly process, which may influence the functional requirements of the mechanism. Most current articles in tolerance analysis of flexible assembly try to predict the consequences of the geometrical defects in the parts on the assembly to be avoided during design stages. Liu and $\mathrm{Hu}$ [1] proposed methods for non-rigid sheet metal assembly are based on linear elastic mechanics based on a model to analyze the effect of deformation and spring-back using finite element methods (FEM), they constructed a sensitivity matrix to establishes the linear relationship between the part variation and the output assembly variation. Another work for Liu and $\mathrm{Hu}$ [2] they findings constitute a new methodology for the tolerancing of deformable parts in series and assembly in parallel. Stricher Alain has two works, his thesis [3] where he proposes strategies for calculating criteria and the calculation cost for the methods of analysis and synthesis of flexible parts tolerances in relation to geometric criteria. To into account, the stiffness variation Stricher proposes in his publication [4] a new approach. Influence Coefficient Method (MIC) was used in [5] for all the steps of the assembling process in order to observe the propagation of the deviations. Dahlström and Lars [6] [7] develop more in the same method (MIC) and they propose a contact algorithm that will be implemented into the method to perform the result. Hugues Favreliere [8] chooses to evaluate the assembly accuracy we address the problem of assembly without games and with games and imposed efforts. Wooyoung [9] proposes a model that extends the concepts of the sources of variation and the Influence Coefficient Method for a compliant mechanical assembly to include the welding distortions. For the same consideration and in the same direction of performance and improvement tolerance analysis of flexible assembly the Influence Coefficient Method was used in a case of Non-linear dimensional variation analysis for sheet metal assemblies [10].

The present work proposes the simulation of the deformable (flexible) mechanisms using the Influence Coefficient Method taking into account the shape defects of each part and its propagation to the assembly of its parts. The performances of this proposed method taking into a count shape defects compared with a simulation without using the effect of shape defects in Influence Coefficient Method.

\section{Influence Coefficient Method}

The Influence Coefficient Method for tolerancing of flexible parts and assemblies corresponds to a method of disturbance where the quantities of interest that will be noted $\alpha j$ as the sum of the products of each contributor, denoted $\delta i$ and of coefficients of influence cij determined by assembly simulations of these parts. The equation shows this approximation in index and matrix notation.

$$
\alpha_{j}=\sum_{i=1}^{N} C_{i j} \delta_{i}
$$

The Influence Coefficient Method supposed linear $C_{i j}$ characterize the influence that the contributor numbered $i$ on the quantity of interest numbered $\mathrm{j}$. The matrix [S] is the "sensitivity matrix"; it 
contains all the coefficients of the influence of the contributor on the quantity of interest.

\section{Shape defects}

To calculate de shape defects for flexible parts, we collect the modes (figure 1) of free sheet metallic whose random amplitudes fallow the distributions laws normal of zero mean and one for standard deviation. After that, the shape defects are generated by superimposition of those modes. For example, we have in figure 2, 3 two examples, the first one for parts without shape defects before and after assembly. The second one for Parts with shape defects before and after assembly.

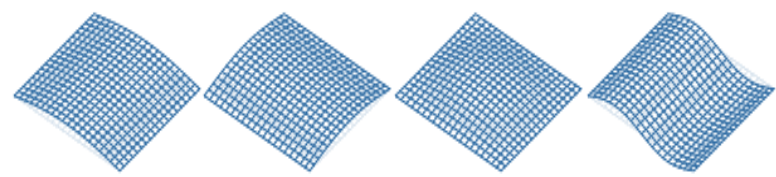

Fig. 1: Uniform shape modes of flat plates

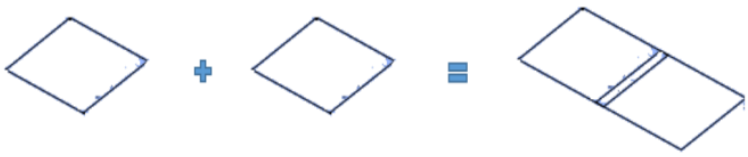

Fig. 2: Parts without shape defects before and after assembly

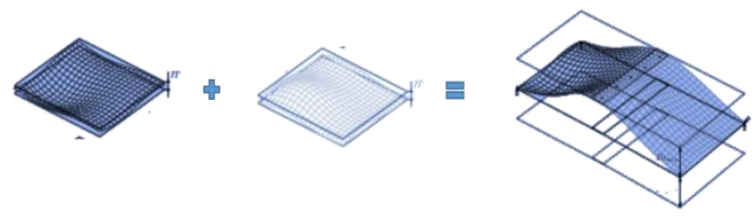

Fig. 3: Parts with shape defects before and after assembly

\section{Influence Coefficient Method with shape defects}

We introduce a method that integrates the shape deviations of the parts to perform the assembly. We model the defect of position and orientation of each part with the representation of the modes Q1 and Q2. Shape defects are parameterized by the following modes, from Q3 Qn. These modes are extracted from the natural modal base of a free-standing sheet metal. These modes are used by Influence Coefficient Method to calculate the surfaces with shape defects. Then, to assemble those parts we have to use our approach which consists in calculates the temporary plan, this calculation is based on the proposed hypothesis of the first contact between the surfaces which is always between the biggest displacement and the smallest and the bridge before the small. After we calculate the temporary plan Position we placed the parts which are aimed at to join on this plan. Then we calculate the distance between all the different assembly points, and with the same hypothesis, we define final plan on which the assembly of the parts considering shape defect deformable geometries will be assembled. The summary of the proposed method is presented in figure 4 below.

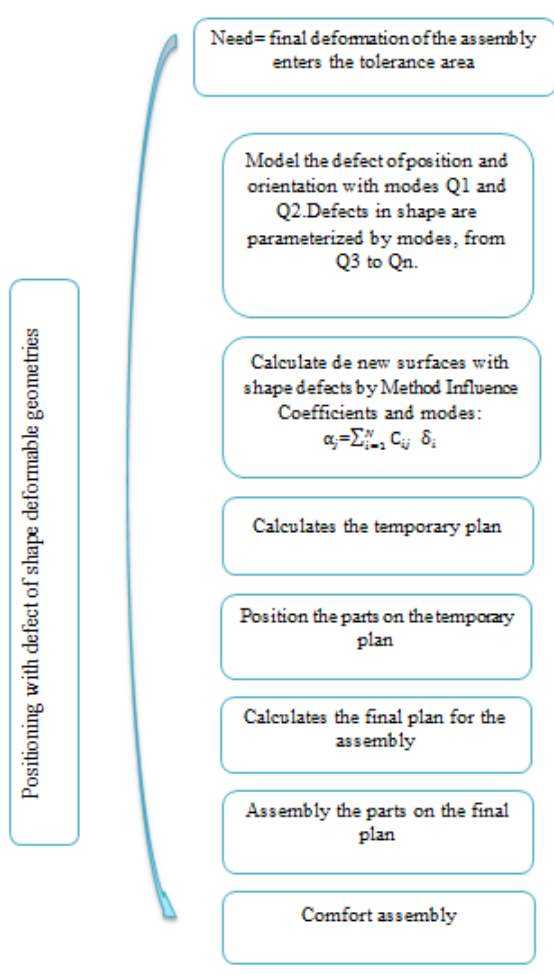

Fig4: The workflow proposal approach for flexible assembly dimensional variation analysis

\section{Assembly study}

For validation of the proposed approach, we did a simulation for an assembly of two parts. The first simulation with MIC including shape defect deformable geometries, and the second is a simulation MIC without shape defect deformable geometries. These simulations intended to perform the prediction of variation of the behavior of flexible parts and assembly. We present in this section an assembly realized with the hypothesis that the surfaces of contact have geometric defects and without geometric defects, orientation and shape on the three dimensions. In the first step, we have two separate parts we apply for each part the source of variation and boundary condition after this step we joined parts to have the assembly final.

- The target: is to analysis flexible parts and assembly with MIC including shape defect deformable geometries, MIC without shape defect deformable geometries.

- Problem: To predict the behavior of flexible parts and assembly to have a modeling near to the real. That is why we need to include with shape defect deformable geometries.

- Hypothesis

- The two plates are assumed to have some initial variation.

- The two plates are considered with shape defect.

- Functional condition: final deformation of the assembly enters the tolerance zone. 


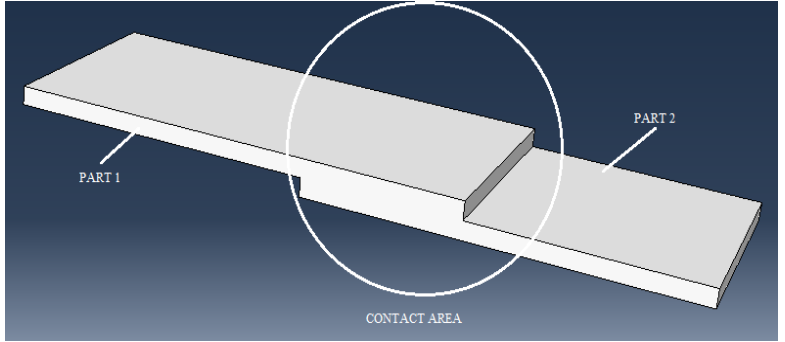

Fig.5: assembly of two flexible plates

\section{Positioning without defect of shape deform- able geometries}

We model the defect of position and orientation of each part with the representation of the modes Q1 and Q2.Defects in shape are parameterized by the following modes, from Q3 Q20 figure 6 . These modes are extracted from the natural modal base of a freestanding sheet metal and boundary condition of each part. The figure presented shows those modes for our parts. Then we use MIC to calculate the profile of the surface including shape defect deformable geometries, the first part is presented in figure 7 and 8 the figure for the second one. After that, we assemble the two parts (figure 9). The result of each of the surfaces 1 and 2 which will be noted by the following S1, S2 respectively. Those surfaces observe in figures 7 and 8 are calculated by Method Influence Coefficients and the combination of the first 20 modes of the spectrum modal to which it is associated. And the next step is to assemble the parts without defect of shape deformable geometries. As you noticed on the figure there is a penetration between the contact surfaces because of the not-considered of the shape defect during the assembly. For this reason, we will try to simulate an assembly with a defect of shape deformable geometries for the second example.
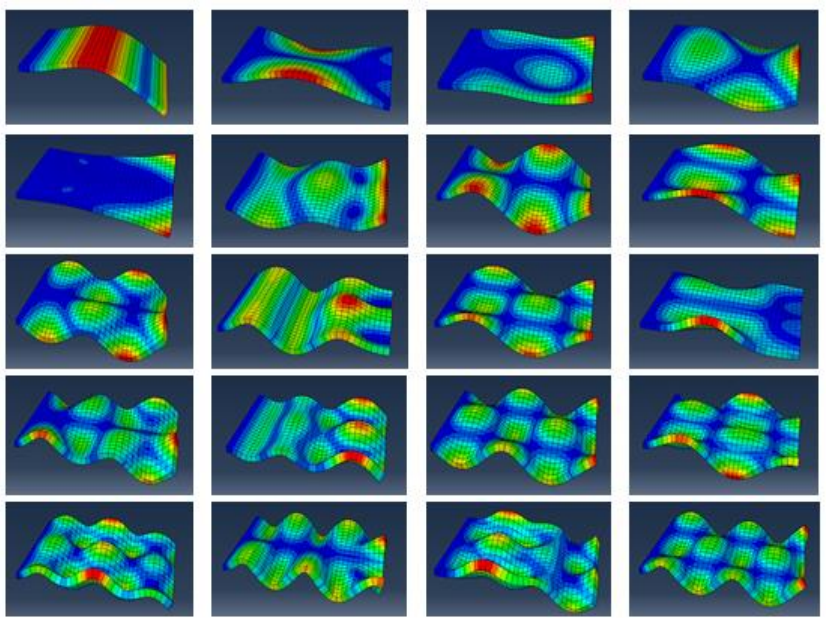

Fig.6: Twenty uniform shape modes of flat plates
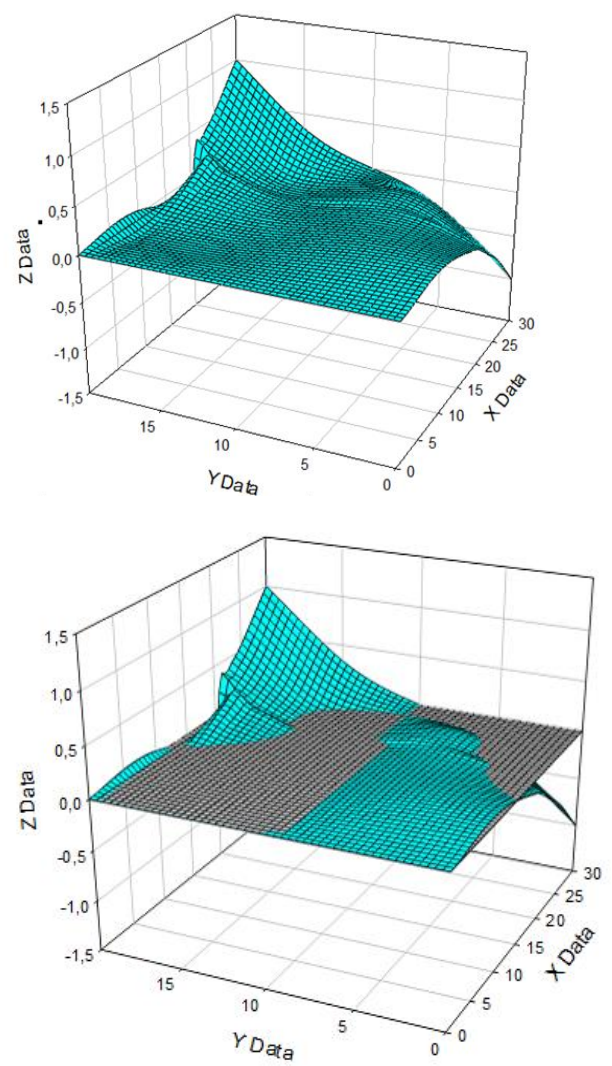

surface $\mathrm{S} 1$ with shape defects

nominal surface S1

Fig.7: Geometric representation of surface S1 with shape defects
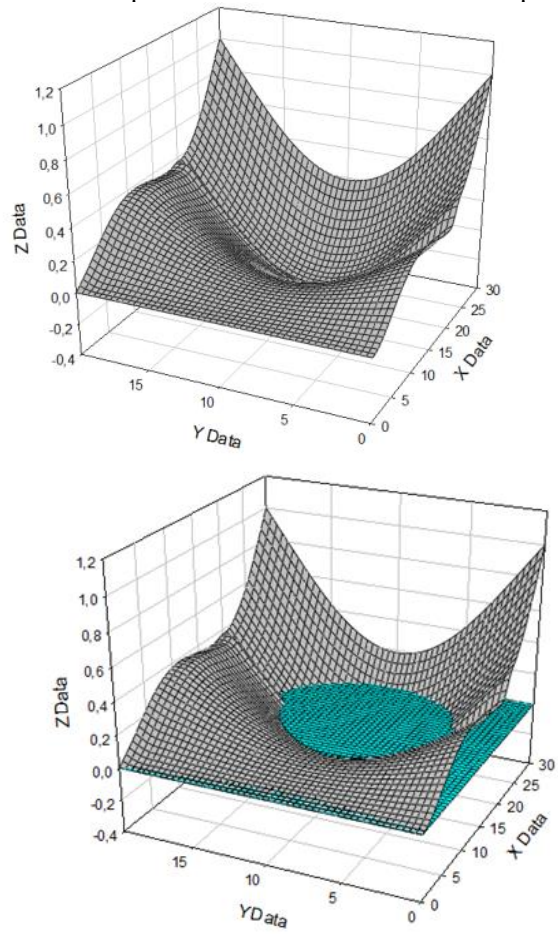

$\square$ nominal surface $\$ 2$

Fig.8: Geometric representation of surface S2 with shape defects 


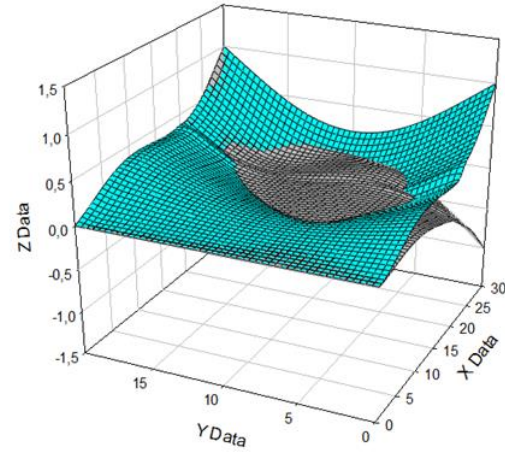

suface $\mathrm{S1}$ with shape defect
suface $\mathrm{S} 2$ with shape defect

Fig.9: Geometric representation of the assembly of surfaces S1 and S2 without shape defects

\section{Positioning with shape defect deformable geometries}

As the first simulation, we are going to the represent the position and orientation defect with the modes Q1 and Q2, and the defects in shape are parameterized by the modes, from Q3 Q20. Just as the first simulation as this simulation the results of the simulation of the modal spectrum and the geometric representation of the defect of each surface are shown in Figures 7 and 8. The result of each of the surfaces S1, S2, and calculated by Influence Coefficient Method and the combination of the first 20 modes of the spectrum. This time, they are the assemblies with taking into account shape defect deformable geometries. And for this reason, we have used our approach which consists in calculates the temporary plan based on the proposed hypothesis of the first contact between the surfaces which is always between the biggest displacement and the smallest and the bridge before the small. We placed the parts which are aimed at to join on the temporary plan. Then we calculate the distance between the different assembly points and with the same hypothesis we Detection of the three points that build the plane of the assembly (figure 10) to define final plan on which the assembly of the parts considering shape defect deformable geometries figure 11 .

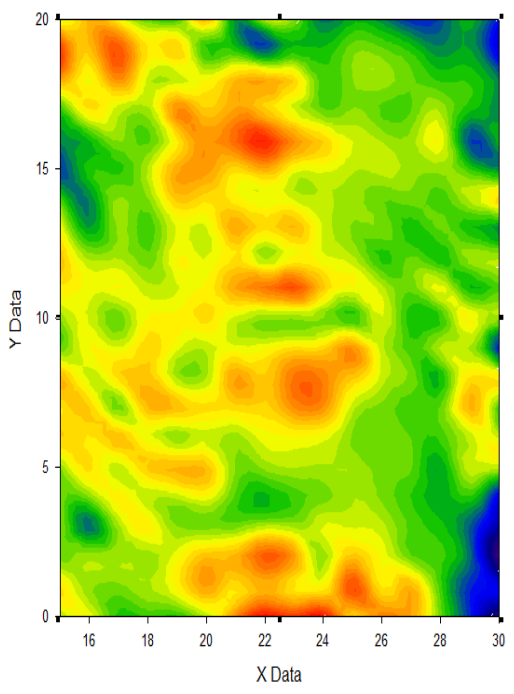

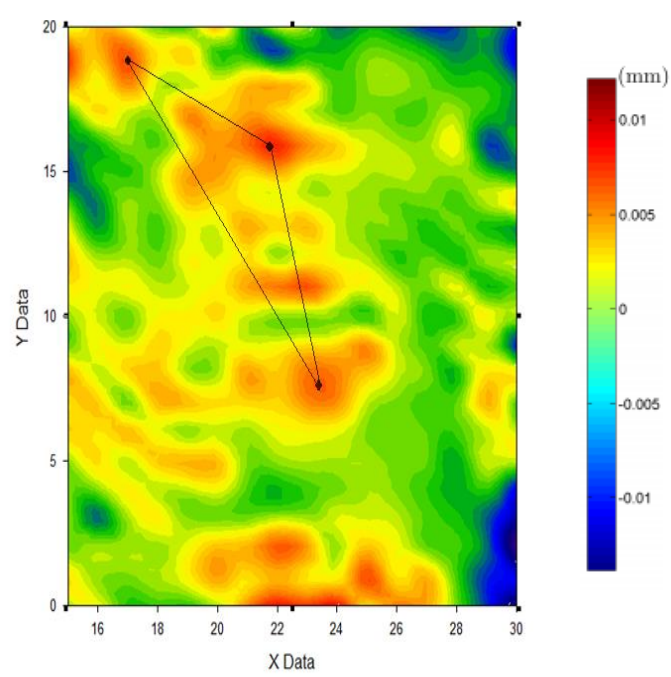

Fig.10: Detection of the three points that build the plane of the assembly
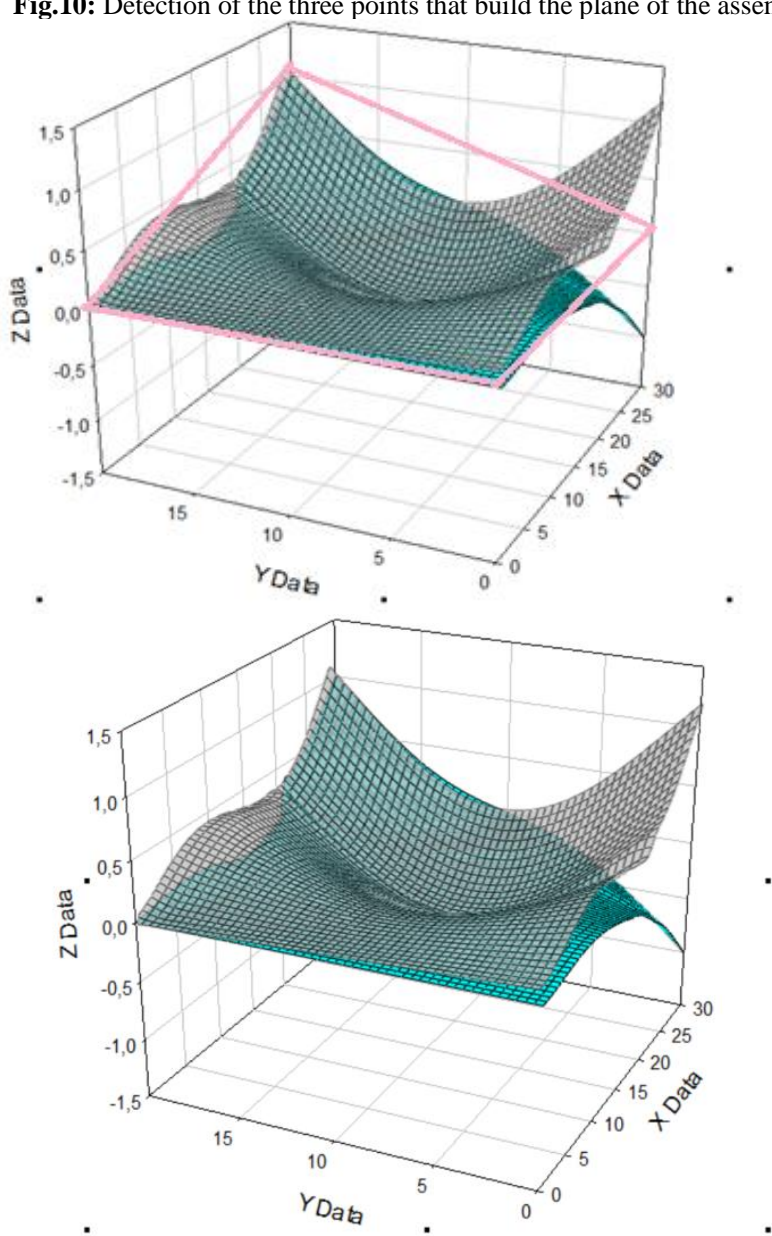

Fig.11: Geometric representation of the assembly of surfaces S1 and S2 with shape defects

\section{Comparison results and discussion}

This comparison shows that when we positioned the parts with shape defect deformable geometries, the assembly is more comfort than when we assembled without shape defect deformable geometries because without taking into account shape defect we risk having pieces penetrates each other. This means that the simulation by Influence Coefficient Method (MIC) with shape defects allows the detecting of the contact area. And more precisely with our approach, we can calculate the assembly plan where we must assemble the plates to have a real assembly and with a great precision. 


\section{Conclusion}

In this paper, we have presented in the first stage an introduction of the method that integrates the shape deviations of the parts to perform the assembly. We model the defect of position and orientation of the part with the natural modal base of a free-standing sheet metal. These modes are used by Influence Coefficient Method to calculate the surfaces with shape defects. Then we use our approach to calculate a temporary plan based on the proposed hypothesis. We placed the parts. After that, we calculate the distance between the different assembly points, and with the same hypothesis, we define final plan on which the assembly of the parts considering shape defect deformable geometries. A simple example of two plates that have been assembled by the new proposed method with shape defect deformable geometries and without defect deformable geometries.

\section{References}

[1] Liu, S. C., and Hu, S. J., 1997. "Variation Simulation for Deformable Sheet Metal Assemblies Using Finite Element Methods". Journal of Manufacturing Science and Engineering, 119(3), Aug., pp. 368-374.

[2] S.C. Liu, S.J. Hu, T.C. Woo, Tolerance analysis for sheet metal assemblies, ASME J. Mech. Des. 118 (1) (1996) 62-67.

[3] Stricher, A., Champaney, L., Thiebaut, F., Fricero, B., and Chevassus, N., 2011. "Mod' ele simplifi ' e pour la simulation d'assemblage de plaques planes avec d' efauts de forme.". In 12 eme Colloque National AIP PRIMECA, no. 1, pp. 1-9.

[4] Stricher.A, "Tolérancement flexible d'assemblages de grandes structures aéronautiques," École normale supérieure de Cachan ENS Cachan, 2013.

[5] Camelio, J. A., Hu, S. J., and Ceglarek, D., 2003. "Modeling Variation Propagation of Multi-Station Assembly Systems With Compliant Parts". Journal of Mechanical Design, 125(4), pp. 673-681.

[6] Dahlstrom .S, L. Lindkvist, "Contact modeling in method of influencecoefficient for variation simulation of sheet metal assemblies", in: Proceedings of IMECE04, 2004 ASME International Mechanical Engineering Congress and Exposition, Anaheim, CA, USA, November 13-20, 2004 (Paper Number: IMECE2004-61550).

[7] Dahlstrom. S, J.S. Hu, R. Soderberg, "Identifying variable effects on the dimensional quality of compliant assembly using computer experiments", in: Proceedings of DETC'02, ASME 2002 Design Engineering Technical Conferences and Computers and Information in Engineering Conference,Montreal, Canada, September 29-October 2, 2002 (Paper Number:DETC2002/DAC-34035).

[8] Hugues Favreliere“" Tolérancement modal : de la méthodologie vers les spécifications" Université de Savoie, 2009.Francais

[9] Wooyoung, C, Hyun, C, "Variation Simulation of Compliant Metal Plate Assemblies Considering Welding Distortion" in Journal of Manufacturing Science and Engineering. Febuary 9, 2015 by ASME.

[10] Xiaoyun , L, Gary Wang,G, “ Non-linear dimensional variation analysis for sheet metal assemblies by contact modeling". Finite Elements in Analysis and Design 44 (2007) pp. 34 - 44. 